\title{
A Single-centre Study of COVID-19 Antibody
}

\author{
Sidra Ghazanfer and Hasan Osman Mahmood \\ Department of Haematology, Col Sultan's Lab, Sialkot Cantt, Pakistan
}

\begin{abstract}
ABSTARCT
Four hundred and fifty-three SARS CoV-2 antibodies tests were conducted using Roche Elecsys ${ }^{\circledR}$ Anti SARS CoV-2 kits (detecting total antibodies) between June 13 to July 8, 2020 (25 days) on Cobas ${ }^{\circledR}$ e411 automatic analyser, based on electrochemiluminescence immunoassay (ECLIA) principle. Samples were collected from walk-in patients at our facility. Among them, 2 (0.4\%) were found equivocal, $289(63.8 \%)$ were found reactive, while $162(35.8 \%)$ were found non-reactive. Moreover, reactive (symptomatic) cases were $262(57.8 \%)$, reactive (asymptomatic) were $27(6.0 \%)$, non-reactive (symptomatic) were $34(7.5 \%)$, while non-reactive (asymptomatic) were 128 (28.3\%). Most common symptom was fever, found in $262(87.9 \%)$ individuals, followed by dry cough $146(49.0 \%)$. Most number of reactive cases, i.e. $110(42.6 \%)$ were those who got themselves tested between 15-21 days after onset of first symptom.
\end{abstract}

Key Words: COVID-19, Coronavirus, Antibodies testing.

How to cite this article: Ghazanfer S, Mahmood HO. A Single-centre Study of COVID-19 Antibody. J Coll Physicians Surg Pak 2021; 31(JCPSPCR):CR55-CR56.

World Health Organization (WHO) labelled COVID-19 as a pandemic on March 11, 2020., ${ }^{1,2}$ The first case in Pakistan was reported by Ministry of Health on February 26, 2020 in Karachi, followed by a second case in Islamabad the same day. ${ }^{3,4}$

As per WHO interim guidelines, laboratory diagnosis of suspected cases should only be done by nucleic acid amplification test (NAAT), such as RT-PCR (an RNA-based molecular test) using the samples commonly taken via nasopharyngeal or oropharyngeal swabs. ${ }^{1,5}$ Tests like viral sequencing and viral cultures are not recommended as routine diagnostic tests, but they do have certain role in researches and further investigations. ${ }^{5}$ On the other hand, antibodies testing is not a diagnostic test. It is only used to check if a person has already had COVID-19; and also to assess the seroprevalence and the immunity status against COVID-19 in a population. ${ }^{1}$

Four hundred and fifty-three walk-in cases were registered in a span of 25 days at a laboratory in Pakistan during 13 June and 8 July, 2020. Brief history of COVID-19 symptoms was taken from patients or their attendants by providing them specially designed proforma (annexure-A). History of fever, dry cough, sore throat, headache, weakness, lethargy, dyspnoea, diarrhoea, anosmia and ageusia (loss of taste) were obtained. Patients were briefed about the phlebotomy procedure, verbal consent was taken and phlebotomy was done using yellow tip cuvette(BD, Germany).

Correspondence to: Dr. Sidra Ghazanfer, Department of Haematology, Col Sultan's Lab, Sialkot Cantt, Pakistan E-mail:dr.sidrahasan@hotmail.com

Received: October 19, 2020; Revised: December 05, 2020; Accepted: February 16, 2021

DOI: https://doi.org/10.29271/jcpsp.2021.JCPSPCR.CR55
Samples were centrifuged to acquire sera as per recommendations by the kit manufacturer. Testing was conducted using FDA approved Elecsys $₫$ Anti SARS-CoV-2 (Roche Holding AG, Basel, Switzerland), run on ECLIA principle-based COBAS $®$ e- 411 analyser. Results were sent for verification via Laboratory Information System (LIS), and verified by the pathologists. The data obtained was incorporated into SPSS V20 (IBM, USA) and analysed. The qualitative data were presented as numbers and percentages.

Among the 453 cases, $2(0.4 \%)$ were found equivocal and were requested to repeat after 10-14 days. The cases that had a patient's value between 0.900 to 1.99 were typed equivocal. Cases that had a patient's value between 2.00 to 4.00 were repeated with a new sample and were typed reactive upon receiving the same or a higher value. Out of remaining 451 cases, 289 (63.8\%) were reactive, while $162(35.8 \%)$ were nonreactive.

We divided the data between symptomatic and asymptomatic cases with further subdivisions, according to their reactive and non-reactive status. In total, four major groups were made, namely: reactive (symptomatic), reactive (asymptomatic), non-reactive (symptomatic) and non-reactive (asymptomatic). This study showed that reactive (symptomatic) cases were 262 $(57.8 \%)$, reactive (asymptomatic) were $27(6.0 \%)$, non-reactive (symptomatic) were 34 (7.5\%), while non-reactive (asymptomatic) were 128 (28.3\%).

It was also observed that among the patients who showed symptoms of the disease, fever was the most common symptom which was found in 262 (87.9\%) individuals, followed by dry cough 146 (49.0\%), weakness 115 (38.6\%), anosmia 101 (33.9\%), ageusia 92 (30.9\%), sore throat 89 (29.9\%), lethargy 
59 (19.8\%), headache 58 (19.5\%), diarrhoea 37 (12.4\%) and dyspnoea 19 (6.4\%) (Table I).

Table I: Frequencies of symptoms in symptomatic patients $(n=296)$.

\begin{tabular}{|l|c|c|}
\hline \multirow{2}{*}{ Symptoms } & \multicolumn{2}{|c|}{ Present } \\
\cline { 2 - 3 } & Frequency & Percent \\
\hline Fever & 262 & $87.9 \%$ \\
\hline Dry cough & 146 & $49.0 \%$ \\
\hline Weakness & 115 & $38.6 \%$ \\
\hline Anosmia & 101 & $33.9 \%$ \\
\hline Ageusia & 92 & $30.9 \%$ \\
\hline Sore throat & 89 & $29.9 \%$ \\
\hline Lethargy & 59 & $19.8 \%$ \\
\hline Headache & 58 & $19.5 \%$ \\
\hline Diarrhoea & 37 & $12.4 \%$ \\
\hline Dyspnoea & 19 & $6.4 \%$ \\
\hline
\end{tabular}

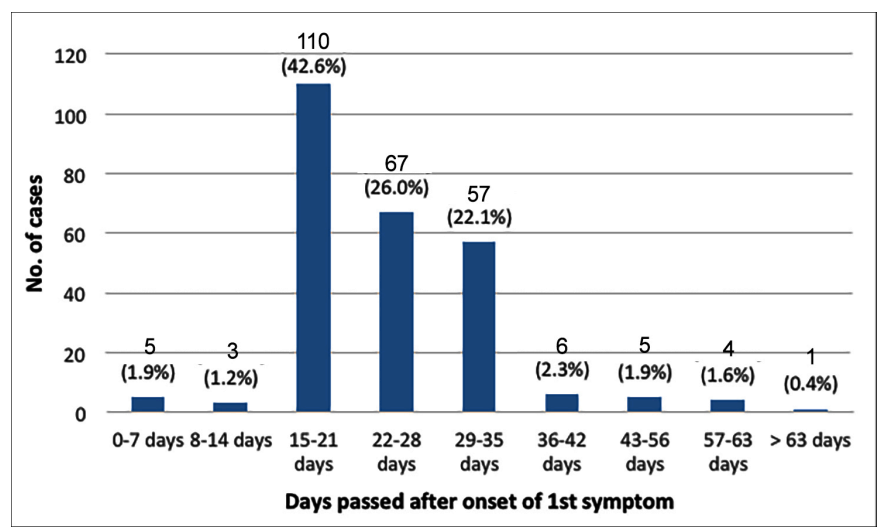

Figure 1: Seroconversion of reactive (symptomatic) cases with respect to days passed after onset of their first symptom $(n=258)$.

At the time of being tested, the individuals were also asked about the days passed after onset of first symptom. It was observed that those who got themselves tested during 15-21 days of symptoms onset, were the highest, i.e. 110 (42.6\%), followed by 67 (26.0\%) who were tested between 22-28 days, and 57 (22.1\%) who were tested between 29-35 days (Figure 1 ). This finding is similar to COVID-19 antibodies behaviour, where IgM becomes positive in $70 \%$ symptomatic patients between 8-14 days, while total antibodies becomes positive in $90 \%$ symptomatic patients between 11-24 days. ${ }^{6}$

This study had a few limitations. The sample size was small as compared to the magnitude of the disease. The cases were all walk-in patients; and therefore, they were combination of severe and non-severe cases, treated and non-treated cases, as well as symptomatic and asymptomatic individuals. Moreover, due to limited resources and decreased testing facilities in this city, most of the cases did not have the privilege of getting RT-PCR done when they first showed the symptoms. Therefore, documented COVID-19 positive cases were only a few. Since total antibodies against SARS-CoV-2 were evaluated; therefore, cases could not be differentiated into acute or chronic stages. Moreover, RT-PCR was not done in any case to confirm negative or positive result.

It was, however, observed in this study that COVID-19 has a wide range of symptoms, with fever being the most common. Not only that, regardless of being acute or chronic, the number of reactive cases shows that a good number of people in the populace may have been infected from COVID-19. A hypothesis can also be made that seroconversion of symptomatic individuals can be observed best between 15-35 days after onset of first symptom. Since this study was done at a very small scale and with very limited resources, further studies on the subject are required to assess the behaviour of the disease by testing antibodies along with RT-PCR of the same individuals.

\section{ACKNOWLEDGEMENT:}

The COO of Col Sultan's Lab, Dr. Col. Sultan Mahmood and the head technician Mr. Syed Abid Hussain Shah are acknowledged for their continuous technical support and help in conducting thestudy.

\section{CONFLICT OF INTEREST:}

The authors declared no conflict of interest.

\section{AUTHORS' CONTRIBUTION:}

SG: Concept, analysis, drafting, interpretation.

HOM: Design of work, data collection, revision of draft

\section{REFERENCES}

1. Rashid ZZ, Othman SN, Samat MNA, Kalsom U, Ken WK. Diagnostic performance of COVID-19 serology assays. Malays J Pathol 2020; 42(1):13-12.

2. Long QX, Liu BZ, Deng HJ, Wu GC, Deng K, Chen YK, et al. Antibody responses to SARS-COV-2 in patients with COVID-19. Nat Med 2020; 26(6):845-8. doi: 10.1038/ s41591-020-0897-1.

3. Saqlain M, Munir MM, Ahmed A, Tahir AH, Kamran S. Is Pakistan prepared to tackle the coronavirus epidemic? Drugs Ther Perspect 2020; 36(5):213-4. doi: 10.1007/ s40267-020-00721-1.

4. Waris A, Atta UK, Ali M, Asmat A, Baset A. COVID-19 outbreak: Current scenario of Pakistan. New Microbes New Infect 2020; 35:100681. doi: 10.1016/j.nmni.2020.100681.

5. Laboratory testing for coronavirus disease 2019 (COVID-19) in suspected human cases: interim guidance [Internet]. World Health Organization. 2020. Available from: http:// apps.who.int/iris/bitstream/handle/10665/331329/WHOCOVID-19-laboratory-2020.4-eng.pdf

6. Beeching NJ, Fletcher TE, Beadsworth MBJ. COVID-19: Testing times. BMJ 2020; 369 :m1403. 